\title{
Core Ossification of Bone Morphogenetic Protein-2-Loaded Collagenated Bone Mineral in the Sinus
}

\author{
Cha, Jae-Kook ; Song, Young Woo ; Kim, Sungtae ; Thoma, Daniel S ; Jung, Ui-Won ; Jung, Ronald E
}

\begin{abstract}
The objective of this study was to investigate in vitro release kinetics and ossification patterns of bone morphogenetic protein-2-soaked collagenated porcine bone mineral (BMP-2/CPBM) in rabbit sinuses. Release kinetics of BMP-2/CPBM was determined in vitro up to 56 days. In 16 rabbits, BMP-2/CPBM (BMP group) and CPBM alone (control group) were bilaterally grafted in both sinuses. After $4(\mathrm{~N}=8)$ and $12(\mathrm{~N}=8)$ weeks, radiographic and histologic analyses were performed. Approximately 40\% of BMP-2 was released from CPBM during 3 days in vitro; release maintained at a reduced level until day 56. In vivo, new bone formation in BMP group was dominant at the center and decreased toward the borders of the sinus, while it mainly possessed close to the sinus membrane and basal bone in control group. At the center, significantly more new bone was found in BMP group compared to control group at 4 weeks $(29.14 \%$ vs. $16.50 \%$; $<<0.05)$. The total augmented volume of BMP group was significantly greater than control group at $4\left(370.13 \mathrm{~mm}^{3}\right.$ vs. $\left.299.32 \mathrm{~mm}^{3}\right)$ and $12(400.40$ $\mathrm{mm}^{3}$ vs. $290.10 \mathrm{~mm}^{3}$ ) weeks $(\mathrm{p}<0.05)$. In conclusion, BMP-2/CPBM demonstrated a core ossification with a greater augmented volume and new bone formation in the center of the sinus compared to CPBM alone. Impact statement The center of the augmented maxillary sinus tends to show a slower and inferior new bone formation compared to the sites near the sinus membrane and basal bone. In this study, bone morphogenetic protein-2 (BMP-2) loaded onto collagenated porcine bone mineral (CPBM) resulted in a greater augmented volume and new bone formation at the center of the grafted sinus compared to CPBM alone. Therefore, BMP-2-added CPBM in maxillary sinus augmentation may potentially be beneficial to the clinicians, in terms of accelerating the new bone formation at the center area where the apical half of the implant fixture usually places.
\end{abstract}

DOI: https://doi.org/10.1089/ten.TEA.2020.0151

Posted at the Zurich Open Repository and Archive, University of Zurich

ZORA URL: https://doi.org/10.5167/uzh-194781

Journal Article

Accepted Version

Originally published at:

Cha, Jae-Kook; Song, Young Woo; Kim, Sungtae; Thoma, Daniel S; Jung, Ui-Won; Jung, Ronald E (2021). Core Ossification of Bone Morphogenetic Protein-2-Loaded Collagenated Bone Mineral in the Sinus. Tissue Engineering. Part A, 27(13-14):905-913.

DOI: https://doi.org/10.1089/ten.TEA.2020.0151 


\section{Core ossification of bone morphogenetic protein-2 loaded collagenated bone mineral in the sinus}

Jae-Kook Cha, DDS, PhD ${ }^{1}$, Young Woo Song, DDS, $\mathrm{PhD}^{1}$, Sungtae Kim, DDS, PhD², Daniel S. Thoma, DMD, $\mathrm{PhD}^{1,3}$, Ui-Won Jung, DDS, $\mathrm{PhD}^{1}$, Ronald E. Jung, DMD, PhD ${ }^{3}$

${ }^{1}$ Department of Periodontology, Research Institute for Periodontal Regeneration, Yonsei University College of Dentistry, Seoul, Korea

${ }^{2}$ Department of Periodontology, School of Dentistry, Dental Research Institute, Seoul National University, Seoul, Korea

${ }^{3}$ Clinic of Reconstructive Dentistry, Center of Dental Medicine, University of Zurich, Zurich, Switzerland

Running title: BMP-2 loaded collagenated bone mineral in the sinus Jae-Kook Cha, DDS, PhD

Department of Periodontology, Yonsei University College of Dentistry

50-1 Yonsei-ro, Seodaemun-gu, Seoul 03722, Republic of Korea.

Tel.: +82-2-22283191

E-mail: chajaekook@gmail.com

Young Woo Song, DDS, PhD

Department of Periodontology, Yonsei University College of Dentistry

50-1 Yonsei-ro, Seodaemun-gu, Seoul 03722, Republic of Korea.

Tel.: $+82-2-22283192$

E-mail: tigger09@naver.com 
Sungtae Kim, DDS, PhD

Department of Periodontology, School of Dentistry, Seoul National University

101 Daehak-ro, Jongno-gu, Seoul 03080, Republic of Korea.

Tel.: +82-2-20724712

E-mail: kst72@snu.ac.kr

Daniel S. Thoma, DMD, PhD

Clinic of Reconstructive Dentistry, Center of Dental Medicine, University of Zurich

Plattenstrasse 11, 8032 Zurich, Switzerland.

Tel.: +41-44-6343311

E-mail: daniel.thoma@zzm.uzh.ch

Ui-Won Jung, DDS, PhD (corresponding author)

Department of Periodontology, Yonsei University College of Dentistry

50-1 Yonsei-ro, Seodaemun-gu, Seoul 03722, Republic of Korea.

Tel.: +82-2-22283185

E-mail: drjew@yuhs.ac 
Ronald E. Jung, DMD, PhD

Clinic of Reconstructive Dentistry, Center of Dental Medicine, University of Zurich

Plattenstrasse 11, 8032 Zurich, Switzerland.

Tel.: +41-44-6343311

E-mail: ronald.jung@zzm.uzh.ch 
Abstract

Objectives: To investigate in vitro release kinetics and ossification patterns of bone morphogenetic protein-2 soaked collagenated porcine bone mineral (BMP-2/CPBM) in rabbit sinuses.

Methods: Release kinetics of BMP-2/CPBM was determined in vitro up to 56 days. In 16 rabbits, BMP-2/CPBM (BMP group) and CPBM alone (control group) were bilaterally grafted in both sinuses. After $4(\mathrm{~N}=8)$ and $12(\mathrm{~N}=8)$ weeks, radiographic and histologic analyses were performed.

Results: Approximately 40\% of BMP-2 was released from CPBM during 3 days in vitro; release maintained at a reduced level until day 56. In vivo, new bone formation in BMP group was dominant at the center and decreased towards the borders of the sinus, while it mainly possessed close to the sinus membrane and basal bone in control group. At the center, significantly more new bone was found in BMP group compared to control group at 4 weeks (29.14\% vs. $16.50 \%$; $p<0.05)$. The total augmented volume of BMP group was significantly greater than control group at 4 (370.13 vs. $299.32 \mathrm{~mm}^{3}$ ) and 12 (400.40 vs. $290.10 \mathrm{~mm}^{3}$ ) weeks $(p<0.05)$.

Conclusion: BMP-2/CPBM demonstrated a core ossification with a greater augmented volume and new bone formation in the center of the sinus compared to CPBM alone.

Keywords: growth factor; xenograft; Schneiderian membrane; bone regeneration; animal study 
Impact statement

The center of the augmented maxillary sinus tends to show a slower and inferior new bone formation compared to the sites near the sinus membrane and basal bone. In the present study, bone morphogenetic protein-2 (BMP-2) loaded onto collagenated porcine bone mineral (CPBM) resulted in a greater augmented volume and new bone formation at the center of the grafted sinus compared to CPBM alone. Therefore, BMP-2-added CPBM in maxillary sinus augmentation may potentially beneficial to the clinicians, in terms of accelerating the new bone formation at the center area where the apical half of the implant fixture usually places. 


\section{Introduction}

A large number of in vivo and clinical studies have demonstrated accelerated and pronounced new bone formation using bone morphogenetic protein-2 (BMP-2) with different carriers in maxillary sinus augmentation for dental implant placement (1-4). However, there are still possibilities to improve the outcome of this procedure from a clinical point of view. Clinically, more than a half of the dental implant length is mainly located in the center of the augmented maxillary sinus, which is distant from the osteogenic sources (nasal bone, sinus membrane). New bone formation, therefore needs to reach the center of the augmented sinus cavity to enable long-term stability of dental implants (5).

To achieve this goal, various biomaterials (e.g. absorbable collagen sponge, hydroxyapatite, beta-tricalcium phosphate) were investigated combined with BMP-2. However it is still unclear which is the most suitable material (6-9). In the case of the maxillary sinus, dimensional stability of the carrier system is considered as one of the critical factors to prevent a potential pneumatization processes (3).

Among the materials used, a block-type bone substitute composed of a bone substitute with a crosslinked collagen matrix could be a suitable carrier due to its structural integrity and characteristics in absorbing and releasing liquid. The collagen component of the material could prevent particle dissipation and provide a scaffold for the ingrowth of regenerative cells (10-12). Moreover, the release of BMP-2 would be sustainably controlled with the collagen and particle component in the block-type graft; BMP-2 soaked in the collagen component would be released initially, and BMP-2 on the surface of the graft particle would be released at a later stage (13). This would potentially improve the bone quality of the augmented sinus gradually by changing the ossifying pattern $(13,14)$, since it is known that new bone formation in a unfavorable and unexpected manner may happen due to an improper dose control of BMP-2 (15).

A few previous in vivo studies have evaluated the efficacy of collagenated block-type bone mineral as a carrier of BMP-2 in the sinus augmentation model. A previous study demonstrated that BMP-2 loaded onto a collagenated bovine bone mineral significantly 
increased the amount of new bone in dog nasal sinus model compared to the control group (2). In particular, a significantly large proportion of newly formed bone was observed in the center of the sinus of the BMP-2 group compared to the control group (44.30 and $5.18 \%$, respectively, $p<0.05$ ). However, this study was the result of observation at 20 weeks in the dog sinus augmentation model, so it was not appropriate to derive the difference of ossifying pattern because bone remodeling was finished at 20 weeks.

Therefore, we hypothesized that the collagenated bone mineral with BMP-2 would show a different ossifying pattern especially in the center of the sinus cavity. Thus, the objective of this study was to investigate (1) the in vitro release kinetics and (2) the ossifying pattern of collagenated porcine bone mineral (CPBM) soaked in BMP-2 at two time points, 4 and 12 weeks, in the rabbit sinus.

\section{Materials and Methods}

\subsection{In vitro release kinetics of BMP-2 from CPBM}

$3.75 \times 10^{-3} \mu \mathrm{g}$ rhBMP-2 (15 $\mu$ l solution at $0.25 \mu \mathrm{g} / \mathrm{ml}$ in buffer; $5 \mathrm{mM}$ glutamic acid, $2.5 \%$ glycine, $0.5 \%$ sorbiotol, $5 \mathrm{mM}$ sodium chloride, $0.01 \%$ tween 80 ) was soak-loaded onto each block of CPBM $(2 \times 2 \times 2 \mathrm{~mm})$ and incubated at room temperature for $30 \mathrm{~min}$. The BMP2/CPBM was then immersed into a 1.5- $\mathrm{ml}$ Eppendorf tube containing $1 \mathrm{ml}$ phosphatebuffered saline. The supernatant from four prepared samples was collected following incubation at $37^{\circ} \mathrm{C}$ for $1,3,6$, and $12 \mathrm{~h}$, and $1,3,7,14,28$, and 56 days. Amount of rhBMP2 in the supernatant was measured using an enzyme-linked immunosorbent assay/ELISA (Human BMP-2, Quantikine, R\&D Systems, Minneapolis, MN, USA).

\subsection{Animals and study design}

Sixteen male New Zealand white adult rabbits (DooYeol Biotech, Seoul, Korea) which weighed 2.5-3.0 kg were used. The experiments were performed according to the guidelines of the Institutional Animal Care and Use Committee of Yonsei Medical Center, Seoul, Korea (Approval No. 2014-0364). The rabbits were housed individually under standard laboratory conditions, and water ad libitum and standard laboratory pellet diet 
were used for feeding according to the ARRIVE guideline (16). The animals were euthanized at 4 and 12 weeks postoperatively ( $n=8$, at each time point).

\subsection{Experimental materials}

The CPBM is a porcine-derived collagenated rigid-type bone block comprising $90-98 \%$ porcine bone mineral and $2-10 \%$ crosslinked collagen. The porosity of CPBM is approximately $77 \%$ (17). The manufacturing process of CPBM was described in previous studies $(18,19)$. In brief, cancellous and cortical porcine bone was processed at $15 \% \mathrm{NaOH}$ for 3 hours to eliminate antigens and placed in boiling water for 72 hours. Then it was mixed with aqueous phase of collagen dispersions. Collagen cross-linking was achieved by dehydrated treatment at $105^{\circ} \mathrm{C}$ under a vacuum of 1 torr for 24 hours. This material has been used in previous clinical trials regarding alveolar bone augmentation $(20,21)$. The CPBM (5 mm in diameter and $10 \mathrm{~mm}$ in length; CollaOss, Bioland, Cheongju, Korea) was soaked 10 minutes either in BMP-2 solution $(0.2 \mathrm{ml}, 0.05 \mathrm{mg} / \mathrm{ml}$; CowellMedi Implant, Busan, Korea) for the BMP group, or in saline for the control group. The dose and concentration of BMP-2 was determined based on previous study (22).

\subsection{Surgical procedure}

The surgical protocol was described in a previous study (23). All procedures were operated under general anesthesia by one experienced surgeon (U.W.J.). In addition, local anesthesia (lidocaine $\mathrm{HCl}$, Huons, Seoul, Korea) was injected at each operated site.

In brief, after an incision along the sagittal line of the nasal bone and reflection of the skin, circular windows ( $\varnothing 5.5-\mathrm{mm}$ ) were bilaterally prepared on the dorsum of both sinuses (Fig. 1) (3). The window disc was carefully removed and stored in saline. The sinus membrane was elevated creating space for the grafting procedure. The experimental biomaterials for each group were placed at both sinuses (one group on each side). In terms of allocation of the groups, the treatments were randomly assigned to the sinuses in the first animal, and then, the left and the right were alternatively switched in the subsequent animals. The removed window discs were positioned on the window to close the entrance. The flaps were primarily closed using 4/0 Monosyn (glyconate absorbable monofilament, B-Braun, Aesculap, Center Valley, PA, USA), and was removed 7 days after the operation. The 
animals received analgesics ( $0.5 \mathrm{mg} / \mathrm{kg}$ ketorolac, Keromin, Hana Pharm, Seoul, Korea) and antibiotics ( $5 \mathrm{mg} / \mathrm{kg}$ Baytril, Bayer Korea) once daily for 5 days. After the healing period of either 4 or 12 weeks, sacrifice of the animals was performed by using overdose of general anesthesia.

\subsection{Sample size}

The required sample size for the experiment was estimated using G Power software (24), with the power of $90 \%$ and the alpha level of 0.10 . According to the previous study performed with the same experimental design which showed significant difference in new bone area at 4 weeks in histomorphometric analysis between the test and control groups $\left(6.16 \pm 2.35 \mathrm{~mm}^{2}\right.$ vs. $\left.3.07 \pm 1.27 \mathrm{~mm}^{2}\right)(13), 8$ rabbits were needed for 4 weeks group and 12 weeks group each, requiring total number of 16 rabbits.

\subsection{Radiographic analysis}

The block specimens of the operated sites and adjacent tissue, which were collected en bloc, were fixed by applying $10 \%$ formalin for 10 days. The block specimens were examined by scanning with micro computed tomography (micro CT; SkyScan 1173, SkyScan, Aartselaar, Belgium) at a resolution of $14.91 \mu \mathrm{m}(130 \mathrm{kV}$ and $60 \mu \mathrm{A})$. 3D image of the maxillary sinus and supporting bone was obtained by reconstructing the micro CT scanned data set with a CT analyzer program (SkyScan) and was visualized with a data viewer program. The total volume of the augmented area (TAV in $\mathrm{mm}^{3}$ ) was calculated based on the previous study (23). Within TAV, the new bone volume (NBV), residual graft material volume (RMV) and the non-mineralized tissue volume were measured.

\subsection{Histologic and histomorphometric analysis}

After decalcifying the block specimens with 5\% formic acid for 14 days, the specimens were embedded in paraffin after dehydration by established routines. Coronal sectioning of the paraffin blocks along the center of the sinuses were performed with the thickness of $5 \mu \mathrm{m}$. Two of the most central sections were stained with hematoxylin-eosin and Masson's trichrome. With the use of optical microscopy (BX50, Olympus, Tokyo, Japan), digital images were obtained from the histologic slides. Histomorphometric measurements were 
also performed in the same way as the previous study (23). The following parameters were measured (in $\mathrm{mm}^{2}$ ):

- Total augmented area (TAA): the area enclosed by the antral bone wall, Schneiderian membrane, and the window.

- Newly formed bone area (NBA): the fraction of newly formed bone within the TAA.

- Residual CPBM area (RMA): the fraction of CPBM within the TAA.

- Fibrovascular area: the fraction of fibrovascular marrow within the TAA.

The augmented area was divided into three specific standardized regions of interest for intragroup comparisons based on the previous study (13): close to the window, at the center, and close to the Schneiderian membrane. The NBA(\%), RMA(\%) and fibrovascular area were measured within a square with standardized size $(1.0 \times 1.0 \mathrm{~mm})$ in each of three regions (Supplement figure 2).

\subsection{Statistical analysis}

The micro-CT and histometric measurements of the samples were analyzed statistically using a SPSS ver. 15.0 (SPSS Inc., Chicago, IL, USA). The Shapiro-Wilk normality test was performed, and all variables were confirmed to be normally distributed. The differences between the BMP group and the control group at the same time period were compared by paired $t$-test, and the differences according to time of healing in each group were compared by independent $t$-test. Statistical significance level was set at $P<0.05$, and the data were shown as means \pm standard deviation values.

\section{Results}

\subsection{In vitro release kinetics}

BMP-2 release exhibited an initial burst (7 days; $50.31 \%$ ) and a gradual decrease up to day 56 (Fig. 2). The total cumulative release of BMP-2 at 56 days amounted to $68.65 \%$, and thus approximately one third of loaded BMP-2 was still retained within the CPBM carrier. 


\subsection{In vivo experiment}

\subsubsection{Clinical observations}

No sinus membrane perforation occurred during the surgical procedure in all animals. There was no unexpected complication including post-operative infection.

\subsubsection{Radiographic analysis}

The 3D-reconstructed images revealed that the window areas were partly closed in all groups. In both groups, the augmented sinus had a dome-shaped appearance filled with radiopaque mineralized tissue without any particle dissipation through sinus membrane (Fig. 3). However, there was a clear difference in the ossifying pattern within the augmented sinus between the BMP and the control group. At 4 weeks, the newly formed bone occupied the center of the augmented sinus like an egg yolk, surrounded by the CPBM particles in the BMP group. On the other hand, in the control group, the newly formed bone was mainly observed near the Schneiderian membrane and window area directly connected with the basal bone. The difference was more evident at 12 weeks. The volume of new bone at the center area was larger and the radiopacity was also increased compared with that at 4 weeks in the BMP group. The particles of the CPBM surrounded the new bone like a shell, while the new bone at the center was directly connected with the adjacent basal bone. These radiographic features were observed in most of the test specimens ( 6 specimens out of 8 in the BMP group; 2 specimens out of 8 in the control group, Supplement figure 1)

The results of radiographic analysis are presented in Table 1. The TAV was significantly greater in the BMP group than the control group (at 4 weeks, $370.1 \pm 47.4$ vs. $299.3 \pm$ $40.27 \mathrm{~mm}^{3}$; at 12 weeks, $400.4 \pm 72.2$ vs. $290.1 \pm 55.3 \mathrm{~mm}^{3}$, respectively, $\left.p<0.05\right)$. The NBV was also significantly greater in the BMP group than the control group at 12 weeks (107.3 \pm 36.4 vs. $\left.74.6 \pm 15.2 \mathrm{~mm}^{3}, p<0.05\right)$.

\subsubsection{Histomorphometric analysis}

At 4 weeks, the collagen matrix, which is a cohesive granule stabilizer in CPBM was not observed in both groups. In the control group, new bone was mainly detected in the original sinus floor area, and barely visible in the central portion of the augmented sinus 
(Fig. 4). At the center region, the remaining bone substitutes were encapsulated by dense fibrotic tissue, and no multinucleated osteoclast-like cells were observed around the particles. In contrast, the BMP group showed a considerable amount of new bone formation throughout the entire sinus cavity with significantly larger size of augmented sinus. In particular, the central portion was filled with a newly formed woven bone and loose connective tissue that contains a large number of blood vessels. Similar to the radiographic findings, the residual particles were mainly distributed on the peripheral part of the augmented sinus, and hardly seen in the central part.

At 12 weeks, the quantity of new bone was increased in both groups (Fig. 5). However, in the control group, there was still a region where a limited amount of new bone was formed in the center portion of the augmented sinus. In the BMP group, completely remodeled new bone was observed throughout the entire sinus cavity.

Table 2 displays the results of the histomorphometric analysis. At 4 weeks, TAA and NBA in the BMP group were significantly greater than those in the control group (TAA, $25.87 \pm$ 4.06 vs. $18.38 \pm 2.04 \mathrm{~mm}^{2}$; NBA, $7.74 \pm 1.09$ vs. $5.15 \pm 1.72 \mathrm{~mm}^{2}$, respectively, $\left.p<0.05\right)$. At 12 weeks, the TAA was comparable to 4 weeks in each group. The NBA was increased in both groups, however there was no difference according to the healing periods.

Along with the histologic appearance, in the central region of the sinus, the NBA(\%) was significantly greater in the BMP group than the control group at 4 weeks (29.14 \pm 5.49 vs. $16.50 \pm 9.04 \%$, respectively, $p<0.05)$. Also, there was a significant difference in the RMA(\%) between the BMP and control groups ( $8.14 \pm 6.84$ vs. $26.00 \pm 12.69 \%$, respectively, $p<0.05)$.

At 12 weeks, the NBA(\%) in the central region was larger in the BMP group than the control group, however, there was no significant difference between the groups ( $p>0.05)$. The BMP group showed significantly smaller value in the RMA(\%) compared to the control group in the central region ( $14.00 \pm 11.77$ vs. $29.00 \pm 9.07 \%$, respectively, $p<0.05)$. The NBA(\%) of the control group was significantly increased according to the healing time in the central region. 


\section{Discussion}

The present study demonstrated that BMP-2 loaded onto CPBM resulted in sustained release kinetics up to 56 days in vitro. Clinically, this led to an increase of the total augmented volume by $30 \%$ (micro-CT) and an increase of new bone formation by $40 \%$ (histology) compared to CPBM alone. Moreover, the BMP group showed characteristics of a 'core ossification' in the majority of the specimens, which was quite different from the physiologic ossification pattern in the control group. This phenomenon was observed at both 4 and 12 weeks. From a clinical point of view, it is believed that the core-dominant new bone formation in the center of the maxillary sinus could enhance osseointegration of the implant body and contribute to long-term stability of dental implants.

The biologic mechanism of the core ossification in the sinus cavity has not been clarified yet. However, this might be explained by the process of rapid post-operative tissue swelling induced by BMP-2 and composition of the CPBM carrier which comprises xenogenetic hydroxyapatite (90\%) and crosslinked collagen matrix (10\%). BMP-2 has been approved for oral maxillofacial reconstruction since 2007, however, it still has several theoretical safety issues including excessive swelling and ectopic bone formation and carcinogenicity (25). Therefore, the FDA recommended not to apply BMP-2 in an off-label usage in 2008.

The tissue swelling during the early healing period is an inevitable biologic response to BMP-2 (26-28). It was reported that the tissue swelling and inflammation after BMP-2 may be due to robust inflammatory reaction as well as sterile seroma and encapsulated hematoma formation (29). This phenomenon was dose-dependent, and it may be advantageous depending on the applied site of BMP-2, if the tissue response is mild and self-limiting. It was demonstrated that this initial postoperative swelling induced a greater initial augmented volume in the BMP group, eventually being replaced by early bone formation in the rabbit sinus augmentation model (13). These results were in line with the present study presenting significantly larger values of augmented volume in the BMP group at 4 weeks. 
The rapid tissue expansion in the present study would be caused by excessive influx of exudate of surrounding tissues, then promoting the migration of CPBM particles to the periphral region of the sinus cavity $(3,13)$. As a result, the collagen matrix dissolved from the CPBM mainly took its place at the center of the augmented sinus. BMP-2 loaded in the collagen matrix was released in a burst, resulting in faster osteoinductive activity at the center area than the BMP-2 loaded in hydroxyapatite at the peripheral. This hypothesis can be confirmed through the result of previous study. Yon and coworkers reported that the osteogenic potential in the maxillary sinus using the porcine bone mineral as a carrier (30). Compared with this study, CPBM used in the present study showed faster release of BMP-2 in the early stage than porcine bone mineral (CPBM $37 \%$ release at 2 hours; Porcine bone mineral $40 \%$ release at 3 days). Moreover, BMP-2 loaded onto porcine bone mineral carrier could not induce the core ossification in the center of the maxillary sinus at 2 weeks, thus it is considered that this would be occurred by collagen in the presnt study.

Furthermore, the crosslinked collagen matrix was used in the present study, which appears to be more prominent in core bone formation because it could remain physically longer than the natural collagen at the center of the augmented sinus $(19,31)$. These results are presumed to be determined by the concentration and adhesion of the collagen matrix loaded on the CPBM, thus further studies with earlier healing period should be needed to clarify its mechanism.

The core ossification was also reflected in the histometric results shown in the center area. In the present study, the NBA(\%) was significantly larger in the BMP group than the control group ( $29.14 \pm 5.49$ vs. $16.50 \pm 9.04 \%$, respectively, $p<0.05$ ), while the RMA(\%) was significantly smaller in the BMP group than the control group (8.14 \pm 6.84 vs. $26.00 \pm$ $12.69 \%$, respectively, $p<0.05)$ at 4 weeks. $N B A(\%)$ of the center in the control group significantly increased at 12 weeks compared to 4 weeks ( $26.50 \pm 6.82$ vs. $16.50 \pm 9.04 \%$, respectively, $p<0.05)$, however, it was still slightly less than the value of the BMP group at 12 weeks ( $26.50 \pm 6.82$ vs. $34.75 \pm 15.79 \%$, respectively, $p>0.05$ ), which implies that BMP loaded CPBM induced faster and more new bone formation especially in the center area. This is considered the most prominent results compared to previous studies. There are several previous studies that described the improved bone formation at the center of the 
augmented sinus by using the BMP-2. It was reported that bone formation was enhanced in the BMP group than the control group at the central region of the augmented porcine bone mineral (14.2 vs. $5.5 \%$, respectively, $p<0.05$ ) (23). Also, another study demonstrated that the significantly greater \%NBA was observed in the center area of the augmented collagenated calcium phosphate in the BMP group at 4 weeks (13). Some studies have attempted to improve bone quality and density in the sinus augmentation with the additional use of small molecules. It was reported that the sodium butyrate embedded in calcium sulfate enhanced osteogenic differentiation and mineralization, especially in the central portion of augmented area showing significantly higher number of osteocalinpositive cells in rabbit sinus. (32)

The hydroxyapatite collagen carrier used in the present study has an osteoconductive potential in the treatment of bone defects with a controlled drug release profile (33). The in vitro release kinetics for BMP-2 in the present study showed a gentle linear profile up to day 56 after an initial burst release. This observation is similar to a previous study using a porcine bone mineral. It was reported that approximately $40 \%$ of BMP-2 was released at day 3, and then gradually decreased until day 21 (23). In the present study, however, there was a greater amount of BMP-2 release at day 3 because of the collagen matrix, and sustained longer till day 56 . These results are promising in terms of sustainability of the drug release when compared with other carrier materials. Eventually, quantity and quality of newly formed bone can be improved (34). In the same vein, Lee et al. reported that the adipogenic induction by BMP-2 released from hydroxyapatite-coated collagen carrier was significantly reduced compared with the collagen alone (35).

CPBM has been used as a carrier for BMP-2 in previous in vivo studies, however there was no studies showing core ossification. It was used for horizontal ridge augmentation in dogs, and bone ingrowth was observed in the BMP-2 group up to the cental region of CPBM. However ossifying pattern could not be confirmed due to the total healing period of 20 weeks (17). Similarly, Kwak et al. transplanted CPBM loaded with BMP-2 into rat fibula to evaluate the osteogenic potential, but at 4 weeks, the new bone was found mainly in the peripheral portion of the CPBM (36). This difference is thought to be a result 
due to the characteristic of the sinus cavity which is completely contained and localized defect used in the present study.

The results of the present study imply some clinical relevance. An additional initial stability of the dental implant can be obtained from the center of the augmented area (sinus). The central portion of the augmented sinus is far from the basal bone or Schneiderian membrane where osteoprogenitor cells are derived $(37,38)$. Hence, it would be valuable to obtain a faster bone formation at the central area of augmented sinus during the early healing stage ( 4 weeks). Moreover, the block-type collagenated hydroxyapatite carrier is easier to clinically manipulate and insert compared to particulated graft materials $(39,40)$.

\section{Conclusion}

Within the limitations of this study, it can be concluded that the addition of BMP-2 to CPBM resulted in a characteristic core ossifying pattern with a greater augmented volume and more new bone formation in the center of the augmented sinus.

\section{Author disclosures}

The authors declare that they have no conflict of interest.

\section{Acknowledgments}

This work was supported by the National Research Foundation of Korea (NRF) grant funded by the Korea government (Ministry of Science, ICT \& Future Planning) (No. NRF2017R1A2B2002537). 


\section{References}

1. Park, J.B. Use of bone morphogenetic proteins in sinus augmentation procedure. J Craniofac Surg 20, 1501, 2009.

2. Cha, J.K., Lee, J.S., Kim, M.S., Choi, S.H., Cho, K.S., andJung, U.W. Sinus augmentation using BMP-2 in a bovine hydroxyapatite/collagen carrier in dogs. J Clin Periodontol 41, 86, 2014.

3. Choi, Y., Lee, J.S., Kim, Y.J., Kim, M.S., Choi, S.H., Cho, K.S., andJung, U.W. Recombinant human bone morphogenetic protein-2 stimulates the osteogenic potential of the Schneiderian membrane: a histometric analysis in rabbits. Tissue Eng Part A 19, 1994, 2013.

4. Kim, M.S., Lee, J.S., Shin, H.K., Kim, J.S., Yun, J.H., andCho, K.S. Prospective randomized, controlled trial of sinus grafting using Escherichia-coli-produced rhBMP-2 with a biphasic calcium phosphate carrier compared to deproteinized bovine bone. Clin Oral Implants Res 26, 1361, 2015.

5. Pjetursson, B.E., Tan, W.C., Zwahlen, M., andLang, N.P. A systematic review of the success of sinus floor elevation and survival of implants inserted in combination with sinus floor elevation. Journal of clinical periodontology 35, 216, 2008.

6. Jung, J.H., Yun, J.H., Um, Y.J., Jung, U.W., Kim, C.S., Choi, S.H., andCho, K.S. Bone formation of Escherichia coli expressed rhBMP-2 on absorbable collagen block in rat calvarial defects. Oral Surgery, Oral Medicine, Oral Pathology, Oral Radiology, and Endodontics 111, 298, 2011.

7. Koempel, J.A., Patt, B.S., O'Grady, K., Wozney, J., andToriumi, D.M. The effect of recombinant human bone morphogenetic protein-2 on the integration of porous hydroxyapatite implants with bone. Journal of Biomedical Materials Research 41, 359, 1998. 
8. Jung, U.W., Choi, S.Y., Pang, E.K., Kim, C.S., Choi, S.H., andCho, K.S. The effect of varying the particle size of beta tricalcium phosphate carrier of recombinant human bone morphogenetic protein-4 on bone formation in rat calvarial defects. Journal of Periodontology 77, 765, 2006.

9. Jung, R.E., Glauser, R., Scharer, P., Hammerle, C.H., Sailer, H.F., andWeber, F.E. Effect of rhBMP-2 on guided bone regeneration in humans. Clinical oral implants research 14, 556, 2003.

10. Blumenthal, N., Sabe, T., andBarrington, E. Healing responses to grafting of combined collagen-decalcified bone in periodontal defects in dogs. Journal of Periodontology 57, 84, 1986.

11. Kasaj, A., Reichert, C., Gotz, H., Rohrig, B., Smeets, R., andWillershausen, B. In vitro evaluation of various bioabsorbable and nonresorbable barrier membranes for guided tissue regeneration. Head \& Face Medicine 4, 22, 2008.

12. Minabe, M., Kodama, T., Kogou, T., Tamura, T., Hori, T., Watanabe, Y., andMiyata, T. Different cross-linked types of collagen implanted in rat palatal gingiva. Journal of Periodontology 60, 35, 1989.

13. Kim, J.S., Cha, J.K., Cho, A.R., Kim, M.S., Lee, J.S., Hong, J.Y., Choi, S.H., andJung, U.W. Acceleration of Bone Regeneration by BMP-2-Loaded Collagenated Biphasic Calcium Phosphate in Rabbit Sinus. Clin Implant Dent Relat Res 17, 1103, 2015.

14. Kang, H.J., Jun, C.M., andYun, J.H. Radiographic and Histologic Evaluation of a Bone Void that Formed After Recombinant Human Bone Morphogenetic Protein-2-Mediated Sinus Graft Augmentation: A Case Report. The International Journal of Periodontics \& Restorative Dentistry 36 Suppl, s151, 2016.

15. Zara, J.N., Siu, R.K., Zhang, X., Shen, J., Ngo, R., Lee, M., Li, W., Chiang, M., Chung, J., Kwak, J., Wu, B.M., Ting, K., andSoo, C. High doses of bone morphogenetic protein 2 induce structurally abnormal bone and inflammation in vivo. Tissue Eng Part A 17, 1389, 2011. 
16. Kilkenny, C., Browne, W., Cuthill, I.C., Emerson, M., andAltman, D.G. Animal research: reporting in vivo experiments: the ARRIVE guidelines. British journal of pharmacology 160, 1577, 2010.

17. Benic, G.I., Joo, M.J., Yoon, S.R., Cha, J.K., andJung, U.W. Primary ridge augmentation with collagenated xenogenic block bone substitute in combination with collagen membrane and rhBMP-2: a pilot histological investigation. Clin Oral Implants Res 28, 1543, 2017.

18. Park, J.I., Yang, C., Kim, Y.T., Kim, M.S., Lee, J.S., Choi, S.H., andJung, U.W. Space maintenance using crosslinked collagenated porcine bone grafted without a barrier membrane in one-wall intrabony defects. J Biomed Mater Res B Appl Biomater 102, 1454, 2014.

19. Cha, J.K., Joo, M.J., Yoon, S., Lee, J.S., Choi, S.H., andJung, U.W. Sequential healing of onlay bone grafts using combining biomaterials with cross-linked collagen in dogs. Clin Oral Implants Res 28, 76, 2017.

20. Lim, H.C., Jung, U.W., You, H., andLee, J.S. Randomized clinical trial of ridge preservation using porcine bone/cross-linked collagen vs. bovine bone/non-cross-linked collagen: cone beam computed tomographic analysis. Clin Oral Implants Res 28, 1492, 2017.

21. Lee, J.-H., Lee, J.-S., Baek, W.-S., Lim, H.-C., Cha, J.-K., Choi, S.-H., andJung, U.-W. Assessment of dehydrothermally cross-linked collagen membrane for guided bone regeneration around peri-implant dehiscence defects: a randomized single-blinded clinical trial. Journal of periodontal \& implant science 45, 229, 2015.

22. Yoon, S.R., Cha, J.K., Lim, H.C., Lee, J.S., Choi, S.H., andJung, U.W. De novo bone formation underneath the sinus membrane supported by a bone patch: a pilot experiment in rabbit sinus model. Clin Oral Implants Res 28, 1175, 2017.

23. Yon, J., Lee, J.S., Lim, H.C., Kim, M.S., Hong, J.Y., Choi, S.H., andJung, U.W. Pre-clinical evaluation of the osteogenic potential of bone morphogenetic protein-2 loaded onto a particulate porcine bone biomaterial. Journal of clinical periodontology 42, 81, 2015. 
24. Faul, F., Erdfelder, E., Lang, A.G., andBuchner, A. G*Power 3: a flexible statistical power analysis program for the social, behavioral, and biomedical sciences. Behav Res Methods 39, 175, 2007.

25. Benglis, D., Wang, M.Y., andLevi, A.D. A comprehensive review of the safety profile of bone morphogenetic protein in spine surgery. Neurosurgery 62, ONS423, 2008.

26. Lu, S.X., Fiorini, T., Lee, J., Prasad, H.S., Buxton, A.N., Bisch, F.C., Dixon, D.R., Susin, C., andWikesjo, U.M.E. Evaluation of a compression resistant matrix for recombinant human bone morphogenetic protein-2. Journal of clinical periodontology 40, 688, 2013.

27. Lee, K.B., Taghavi, C.E., Murray, S.S., Song, K.J., Keorochana, G., andWang, J.C. BMP induced inflammation: A comparison of rhBMP-7 and rhBMP-2. J Orthop Res 30, 1985, 2012.

28. Woo, E.J. Recombinant human bone morphogenetic protein-2: adverse events reported to the Manufacturer and User Facility Device Experience database. The Spine Journal 12, 894, 2012.

29. Lee, K.B., Taghavi, C.E., Song, K.J., Sintuu, C., Yoo, J.H., Keorochana, G., Tzeng, S.T., Fei, Z., Liao, J.C., andWang, J.C. Inflammatory characteristics of rhBMP-2 in vitro and in an in vivo rodent model. Spine (Phila Pa 1976) 36, E149, 2011.

30. Yon, J., Lee, J.S., Lim, H.C., Kim, M.S., Hong, J.Y., Choi, S.H., andJung, U.W. Pre-clinical evaluation of the osteogenic potential of bone morphogenetic protein-2 loaded onto a particulate porcine bone biomaterial. Journal of clinical periodontology 42, 81, 2015.

31. Jung, U.W., Cha, J.K., Vignoletti, F., Nuñez, J., Sanz, J., andSanz, M. Simultaneous lateral bone augmentation and implant placement using a particulated synthetic bone substitute around chronic peri-implant dehiscence defects in dogs. Journal of clinical periodontology 44, 1172, 2017.

32. Cha, J.K., Lim, H.C., Lee, J.S., Choi, S.H., Woo, K.M., andJung, U.W. Sinus augmentation using a histone deacetylase inhibitor in a calcium sulfate carrier in rabbit: A pilot study. Journal of biomedical materials research Part B, Applied biomaterials 105, 1916, 2017. 
33. Dou, D.D., Zhou, G., Liu, H.W., Zhang, J., Liu, M.L., Xiao, X.F., Fei, J.J., Guan, X.L., andFan, Y.B. Sequential releasing of VEGF and BMP-2 in hydroxyapatite collagen scaffolds for bone tissue engineering: Design and characterization. Int J Biol Macromol 123, 622, 2019.

34. Agrawal, V., andSinha, M. A review on carrier systems for bone morphogenetic protein2. J Biomed Mater Res B Appl Biomater 105, 904, 2017.

35. Lee, J.S., Kim, T.W., Park, S., Kim, B.S., Im, G.I., Cho, K.S., andKim, C.S. Reduction of Adipose Tissue Formation by the Controlled Release of BMP-2 Using a HydroxyapatiteCoated Collagen Carrier System for Sinus-Augmentation/Extraction-Socket Grafting. Materials (Basel) 8, 7634, 2015.

36. Kwak, E.J., Cha, I.H., Nam, W., Yook, J.I., Park, Y.B., andKim, H.J. Effects of locally administered rhBMP-2 and bisphosphonate on bone regeneration in the rat fibula. Oral Dis 24, 1042, 2018.

37. Jakob, M., Hemeda, H., Janeschik, S., Bootz, F., Rotter, N., Lang, S., andBrandau, S. Human nasal mucosa contains tissue-resident immunologically responsive mesenchymal stromal cells. Stem Cells Dev 19, 635, 2010.

38. Cho, K.S., Park, H.Y., Roh, H.J., Bravo, D.T., Hwang, P.H., andNayak, J.V. Human ethmoid sinus mucosa: a promising novel tissue source of mesenchymal progenitor cells. Stem Cell Res Ther 5, 15, 2014.

39. Orsini, G., Scarano, A., Piattelli, M., Piccirilli, M., Caputi, S., andPiattelli, A. Histologic and ultrastructural analysis of regenerated bone in maxillary sinus augmentation using a porcine bone-derived biomaterial. Journal of Periodontology 77, 1984, 2006.

40. Ramirez-Fernandez, M.P., Calvo-Guirado, J.L., Delgado-Ruiz, R.A., del Val, J.E.M.S., Vicente-Ortega, V., andMeseguer-Olmos, L. Bone response to hydroxyapatites with open porosity of animal origin (porcine [OsteoBiol (R) $\mathrm{mp} 3$ ] and bovine [Endobon (R)]): a radiological and histomorphometric study. Clinical oral implants research 22, 767, 2011. 
Correspondence address:

Ui-Won Jung, DDS, PhD

Department of Periodontology, Yonsei University College of Dentistry

50-1 Yonsei-ro, Seodaemun-gu, Seoul 03722, Republic of Korea.

Tel.: $+82-2-22283185$

Fax: $+82-2-3920398$

E-mail: drjew@yuhs.ac 
Figure legend

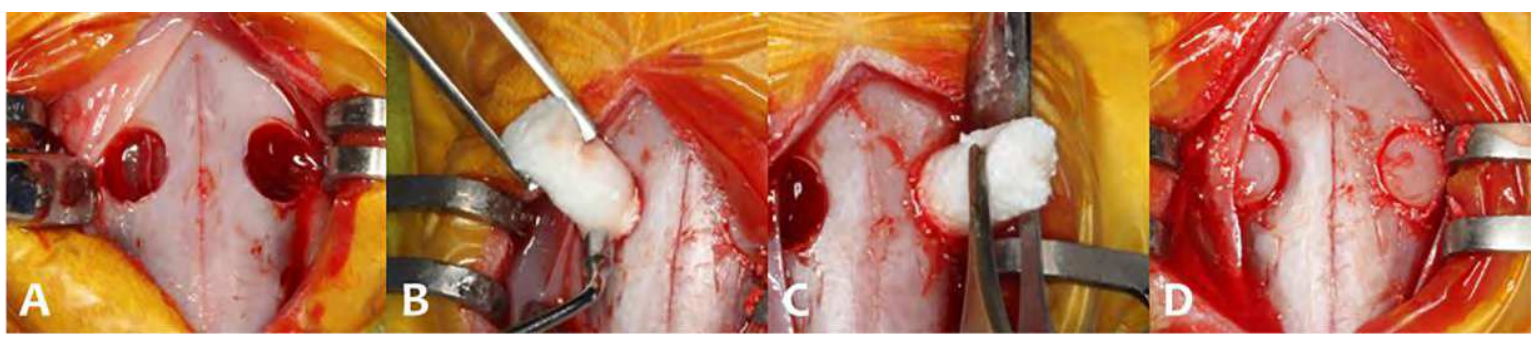

Figure 1. Clinical photographs of the surgical procedure. Circular windows of $5.5 \mathrm{~mm}$ diameter removed state on both sides (A). Applying rhBMP-2 soaked CPBM at one side (B) and saline soaked CPBM at contralateral side (C). Covering the grafted sites with cortical windows on both sides (D). 


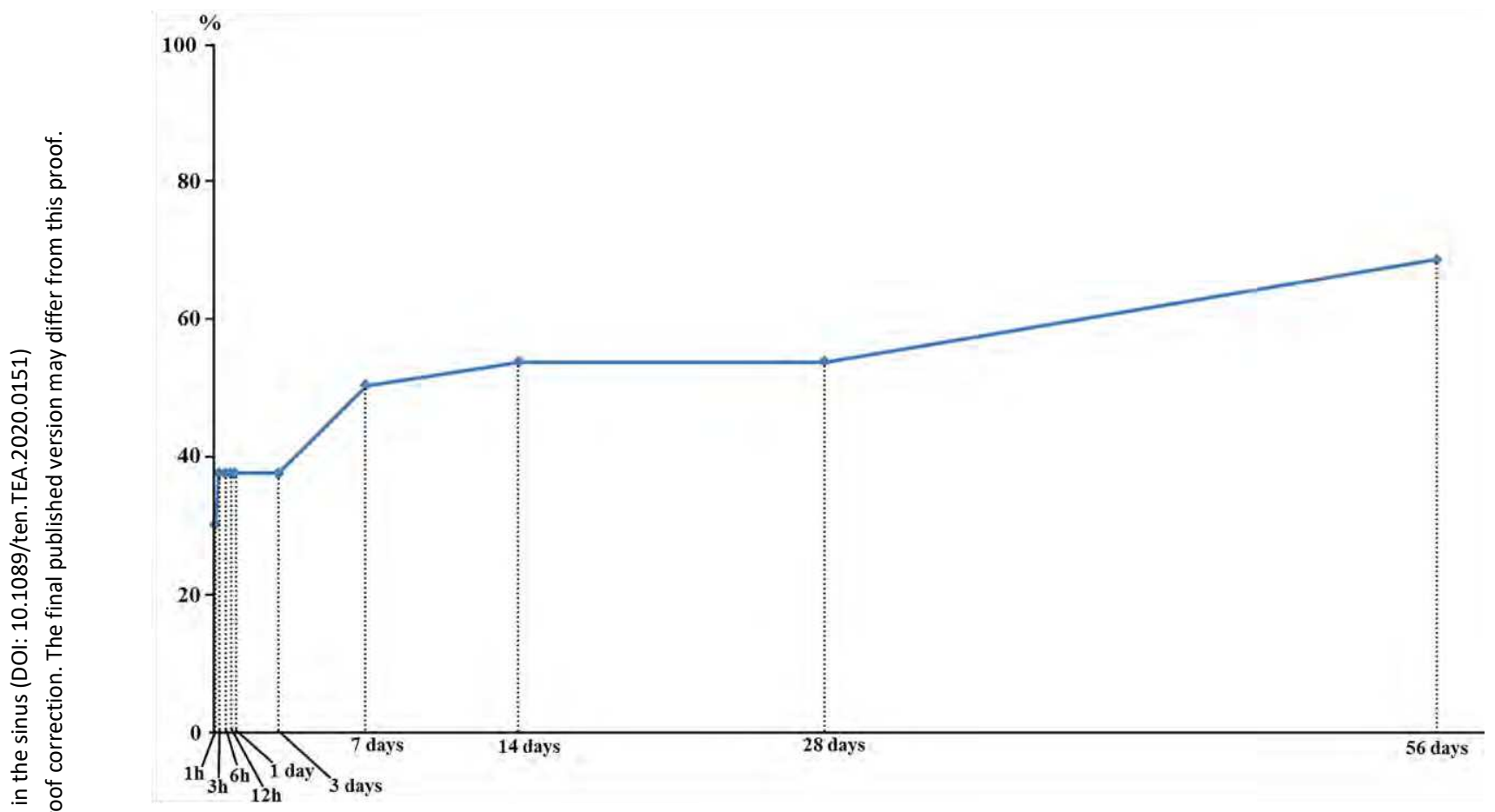

Figure 2. Graphic representation of in vitro experiment for confirming releasing kinetics of bone morphogenetic protein-2 (BMP-2) soaked collagenated porcine bone mineral (CPBM). The releasing rate of BMP-2 gradually decreased from day 7 to day 56 , compared to the initial increase observed until day 7. 


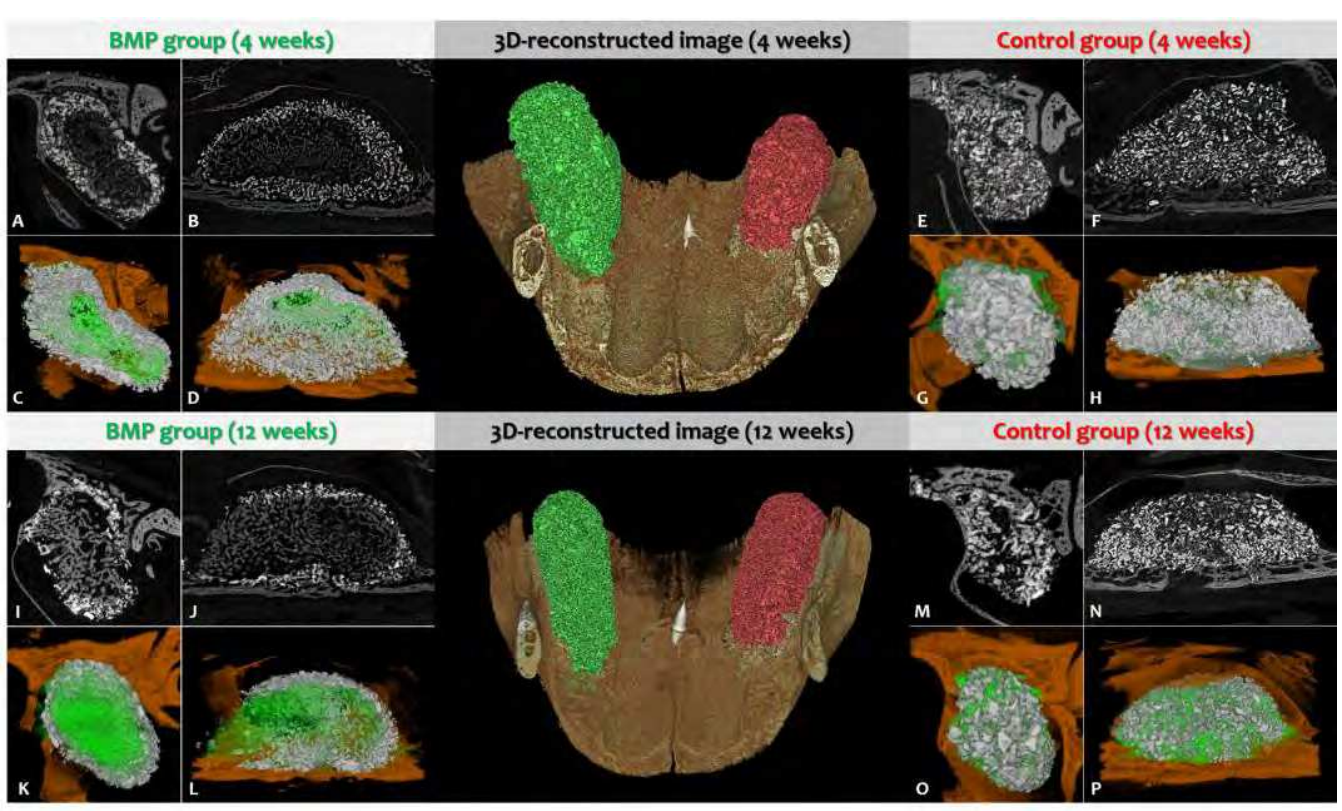

Figure 3. Representative micro-CT images. Three dimensionally (3D) reconstructed microCT images of 4 weeks (upper) and 12 weeks (lower) are shown in the center column, of which the green and red colors indicate BMP group and control group, respectively. Images in the left and right columns represent the results in BMP group and control group, respectively. In colored images, green color and white color present newly formed bone and residual graft material particles, respectively $(C, D, G, H, K, L, O, P)$. Core-dominant new bone formation was observed in BMP group at 4 weeks $(A, B, C, D)$ and 12 weeks $(I, J$, $K, L)$. In control group, new bone formation mainly took place near the window and membrane at 4 weeks $(E, F, G, H)$ and 12 weeks $(M, N, O, P)$. 

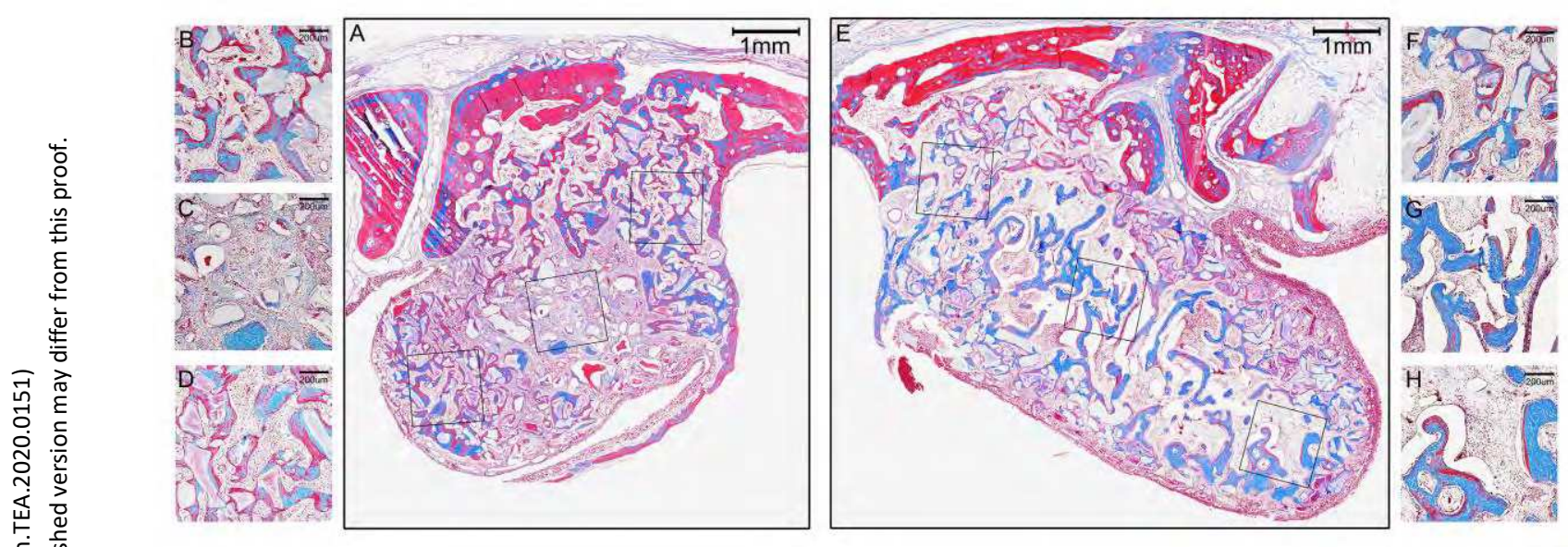

Figure 4. Descriptive histology at 4 weeks of healing. Black squares ( $1 \mathrm{~mm} \times 1 \mathrm{~mm}$ in size) shown in the slides with low magnification ( $A$ and $E$ ) ( $x 20$, scale bar $=1 \mathrm{~mm}$ ), indicate the region of interests (ROI) at three different levels, which are the membrane, center, and window, and they were highly magnified $(x 100$, scale bar $=200 \mu \mathrm{m})$ for further investigation (B-D, F-H). Well-maintained augmented sites are observed in both the BMP group (A) and the control group (E). In the BMP group, newly formed bone (NB) was dominant with less amount of residual graft material (RM) in the center (C), while RM surrounded by NB could be easily seen in the areas near the membrane (B) and the window (D). On the other hand, in the control group, NB and RM were mainly present near the membrane $(F)$ and the window $(H)$, and the center area showed RM encapsulated by dense connective tissue and hardly seen NB (G). NB, new bone; RM, residual bone graft material; FT, fibrous tissue. 

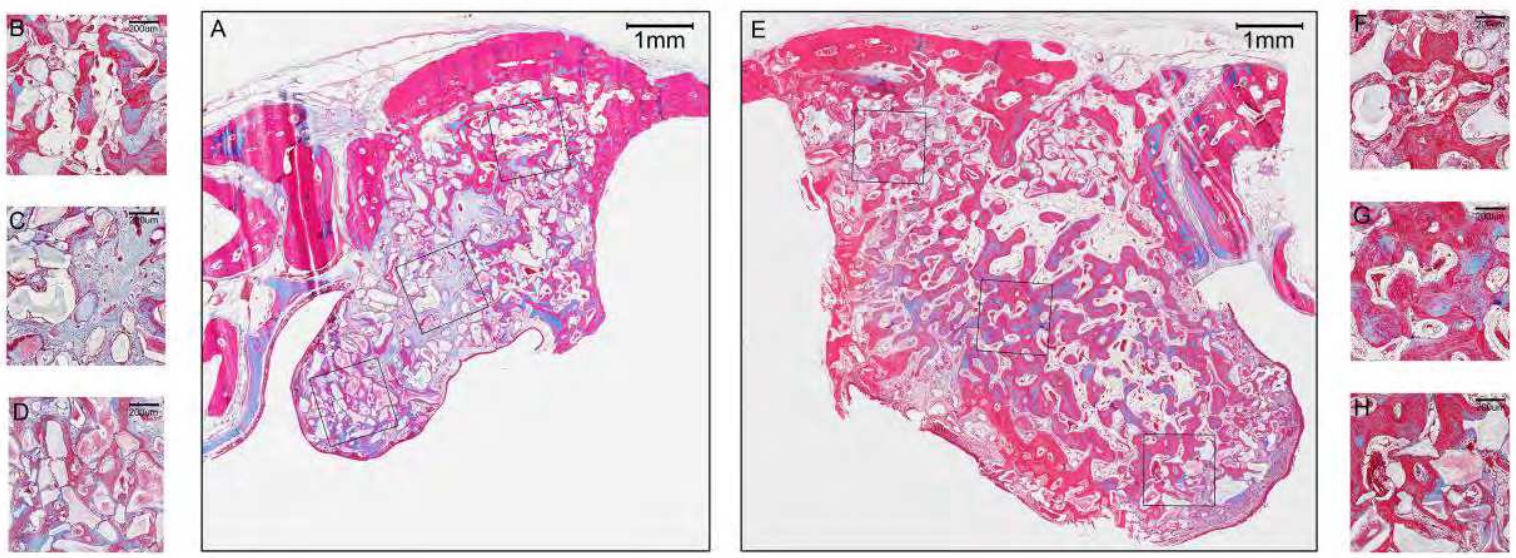

Figure 5. Descriptive histology at 12 weeks of healing. Just as in the figure 5, the black squares in the slides with low magnification $(A$ and $E)(x 20$, scale bar $=1 \mathrm{~mm})$ indicate the region of interests (ROI) near the membrane, in the center, and near the window, and they were magnified $(B-d, F-H)(\times 100$, scale bar $=200 \mu \mathrm{m})$. Increased amount of NB was found in each group compared to 4 weeks, however there was more NB observed in the BMP group $(A)$ compared to the control group $(E)$ throughout the entire augmented sinuses. In BMP group, NB was increased evenly in all three ROIs (B, C, D), while in control group, still the NB was mainly observed near the membrane $(F)$ and the window $(H)$ and less NB was found in the center (G). NB, new bone; RM, residual bone graft material; FT, fibrous tissue. 
Table 1. Results of the radiographic analysis (mean \pm standard deviation)

\begin{tabular}{|c|c|c|c|c|c|}
\hline $\begin{array}{l}\text { Healing } \\
\text { periods }\end{array}$ & Group & $\operatorname{TAV}\left(\mathrm{mm}^{3}\right)$ & $\mathrm{NBV}\left(\mathrm{mm}^{3}\right)$ & $\mathrm{RMV}\left(\mathrm{mm}^{3}\right)$ & $\begin{array}{l}\text { The others } \\
\qquad\left(\mathrm{mm}^{3}\right)\end{array}$ \\
\hline \multirow[t]{2}{*}{4 weeks } & BMP & $370.13 \pm 47.42^{\mathrm{a}}$ & $107.41 \pm 29.13$ & $42.82 \pm 4.90$ & $216.29 \pm 51.78^{\mathrm{a},+}$ \\
\hline & Control & $299.32 \pm 40.27^{a}$ & $94.57 \pm 20.48$ & $44.69 \pm 9.58$ & $156.80 \pm 27.58^{a}$ \\
\hline \multirow[t]{2}{*}{12 weeks } & BMP & $400.40 \pm 72.16^{a}$ & $107.28 \pm 36.44^{a}$ & $41.49 \pm 7.47$ & $248.51 \pm 53.83^{a,+}$ \\
\hline & Control & $290.10 \pm 55.27^{a}$ & $74.56 \pm 15.20^{\mathrm{a}}$ & $45.12 \pm 12.05$ & $165.66 \pm 52.87^{\mathrm{a}}$ \\
\hline \multicolumn{6}{|c|}{${ }^{a}$ (bold): Significantly different between the two group at the same observation period $(p<$} \\
\hline \multicolumn{6}{|l|}{$0.05)$} \\
\hline \multicolumn{6}{|c|}{${ }^{+}$(bold): Significantly different between the two time points within the same group ( $p<$} \\
\hline 0.05$)$. & & & & & \\
\hline
\end{tabular}


Table 2. Results of the histomorphometric analysis (mean \pm standard deviation)

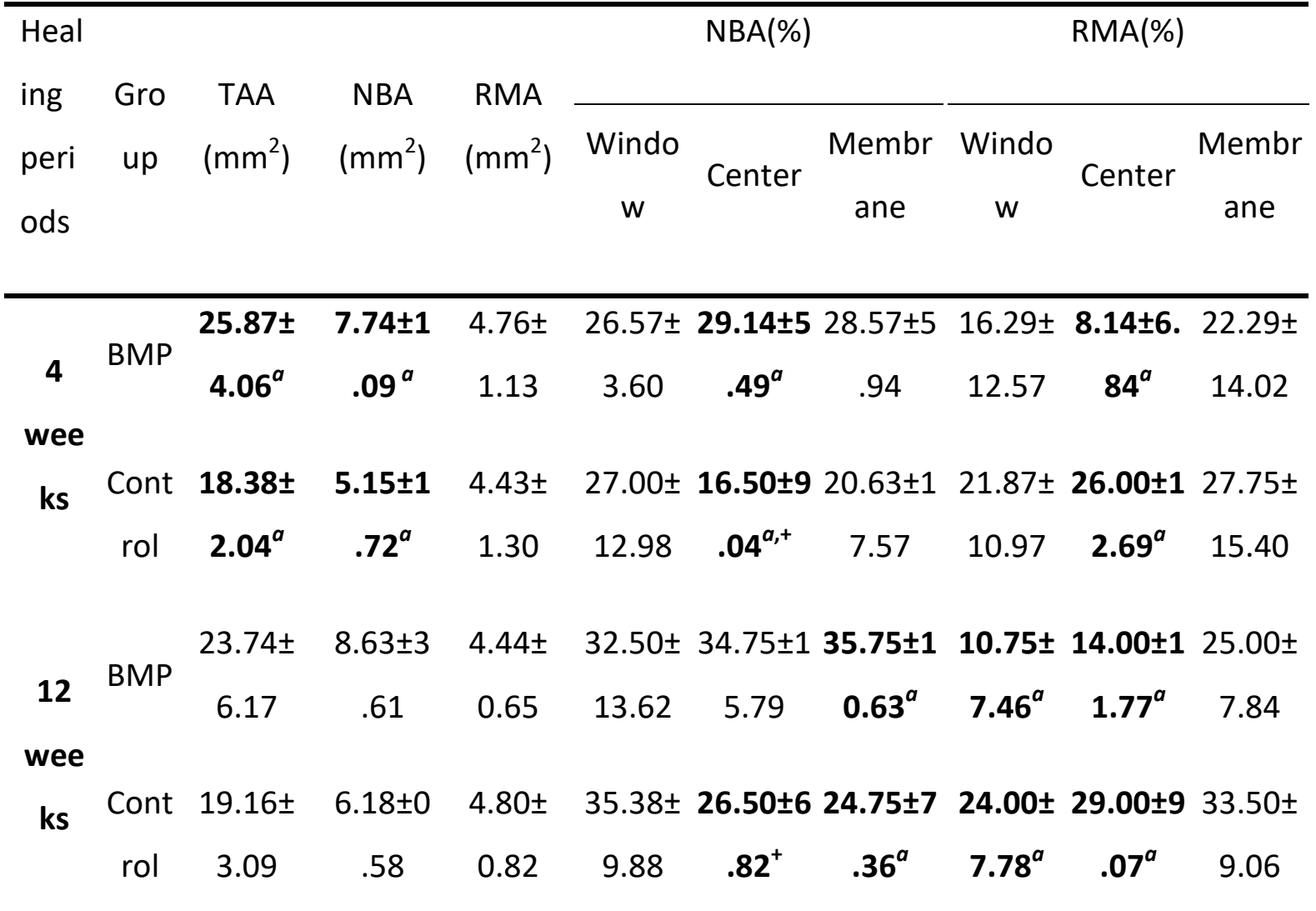

${ }^{\mathrm{a}}$ (bold): Significantly different between the two group at the same observation period $(p<$ $0.05)$.

${ }^{+}$(bold): Significantly different between the two time points within the same group $(p<$ $0.05)$.

(TAA, total augmented area; NBA, new bone area; RMA, residual material area; NBA(\%), fraction of new bone within a standardized region of interest; RMA(\%) fraction of residual material within a standardized region of interest) 